Bol. Acad. peru. leng. 64. 2018 (111-126)

\title{
MÁS VALE ARTÍCULO EN MANO QUE VER UN CIENTO VOLAR. CONTRIBUCIÓN DE LA LITERATURA A LA ESCRITURA DE ARTÍCULOS CIENTÍFICOS
}

\section{A PAPER IN THE HAND IS WORTH TWO IN THE BUSH. CONTRIBUTION OF LITERATURE TO THE PRODUCTION OF SCIENTIFIC PAPERS}

\author{
Rafael Cartay \\ Universidad Técnica de Manabí (UTM) \\ Universidad de los Andes (ULA)
}

\section{Resumen:}

Los participantes a los talleres de redacción de artículos científicos plantean con frecuencia inquietudes en relación con la escritura de las distintas secciones de la estructura del artículo: título, resumen, introducción, metodología, contenido y discusión. Como los hallazgos científicos deben ser transmitidos con claridad, brevedad y en un estilo coherente, planteo que los científicos interesados en transmitir con propiedad tales conocimientos se beneficien de la ayuda de los escritores, cuyo propósito principal es el de transmitir al lector de manera clara y amena las descripciones de paisajes, los estados de ánimo y los pensamientos y acciones de sus personajes de ficción. 


\section{Abstract:}

Participants in scientific research paper writing workshops often raise concerns regarding the different sections of the structure of the paper: title, abstract, introduction, methodology, content, and discussion. Since scientific findings must be conveyed clearly, briefly, and in a coherent style, I propose that scientists interested in properly transmitting such knowledge benefit from the help of writers, whose primary purpose is to convey to the reader, in a clear and enjoyable manner, the descriptions of landscapes, moods, and the thoughts and actions of their fictional characters.

Palabras clave: redacción de artículos; estilo de escritura; literatura y comunicación científica.

Key words: research paper writing, writing style, literature and scientific communication.

Fecha de recepción: $01 / 08 / 2018$

Fecha de aceptación:

$31 / 10 / 2018$

«Para escribir, hay que atreverse...Hay que seguir atreviéndose, porque solo escribiendo se aprende a escribir. Y escribiendo mucho. Y leyendo mucho».

Di Marco \& Pendzik, 2013: 33.

\section{Introducción}

La evolución continua de la ciencia ha multiplicado sus hallazgos desde principios del siglo XX a un ritmo sin precedentes en la historia humana, 
haciendo necesario el desarrollo de sistemas de información para organizar y transmitir esos conocimientos. Una de las maneras de hacerlo, probablemente la más conocida, es la que se conoce como formato IMRYD, siglas que compendian la secuencia de pasos relacionados con la investigación que conduce al hallazgo científico. En ese formato, I significa introducción (¿qué problema o cuestión se aborda?); M, metodología o método de abordaje (¿cómo se estudia el problema?); $\mathrm{R}$, resultados (¿cuál fue el hallazgo?) y $\mathrm{D}$, discusión (¿qué significan esos resultados?). La lógica implícita en ese formato es simple: ayudar al expositor-investigador para que organice su trabajo y sus ideas con claridad, y logre transmitir adecuadamente a su lector el conocimiento derivado de esa experiencia o indagación científica.

\section{Metodología}

Los manuales utilizados para enseñar la técnica IMRYD son muchos (unos más claros que otros aunque todos se repiten). En este artículo me valdré de ellos en lo esencial, buscando dar algunas claves para responder a las preguntas más frecuentes planteadas en los talleres de escritura de artículos científicos. Esta es una pequeña investigación documental y descriptiva, que consiste en la revisión de una selecta bibliografía básica sobre el tema, sin mayor pretensión que la de transmitir al lector mi experiencia particular como investigador. Examinaré todas las partes del artículo, aunque pondré el énfasis sobre la introducción y los resultados para mostrar dos estilos distintos: el científico y el literario. Los compararé para destacar sus parecidos y presentar cómo los literatos pueden contribuir con los científicos a expresarse con mayor soltura y claridad.

\section{Resultados}

¿Qué significa y cómo se crea un buen título?

El título es la puerta de entrada de un artículo científico y la tarjeta de presentación del autor. Es una puerta que debe estar bien señalada para que el lector traspase su umbral y entre a la casa. García Márquez decía que «Es más fácil atrapar a un conejo que a un lector». 
iCuántos lectores se han quedado afuera porque la puerta les resulta inhóspita y la invitación fría! Si el título es confuso y sin fuerza, nadie se animará a leer tu artículo. El marketing alimentario recomienda dar a probar una muestra de un producto atractivo al consumidor para que lo adquiera. La función del título es atraer potenciales lectores a tu artículo dándoles una muestra inteligente que describa tu propuesta (título descriptivo) o tu hallazgo (título informativo). Un título debe ser conciso, aparte de atractivo. Debe tener de doce a quince palabras. No más. Lo dicen todos los manuales, sin decir por qué. Creo que es una buena medida: la mitad de los caracteres promedio de un Twitter o cerca del número promedio que cabe en una línea cuando escribes. $\mathrm{Ni}$ tan calvo ni con dos pelucas.

Un título jamás debe tener errores gramaticales ni incorrecciones del lenguaje. No debe llevar abreviaturas, ni muchas preposiciones, ni faltas ortográficas. Debe evitar los signos de admiración o de interrogación, el punto y coma. O huir de entradas como aspectos de, comentarios sobre, estudio de, observaciones de, resultados de una investigación sobre, etc. No es fácil. Las hemos usado mucho. Lo interesante es apartarse de la rutina y descartar expresiones útiles, pero simplistas. Brailovsky (2000) propuso cincuenta expresiones del lenguaje académico que resultan útiles para componer un artículo científico. Son como piezas de un rompecabezas que uno inserta en el texto en la medida en que sean necesarias. Por ejemplo, si quieres hacer una crítica, escribes: «La principal crítica a esa posición se centra en...». Si quieres presentar unas conclusiones: "Concluyo que, tras la revisión efectuada, ...». Si deseas presentar la idea de otros: «Pérez (2016) sostiene que...». Pero si presentas una idea propia, anotas: «Sostengo que...». Todas esllas son frases hechas, útiles para copiar y pegar, a menos que seas Tabucchi, quien escribió la novela Sostiene Pereira para darle propiedad al verbo sostener, ese verbo casi ingrávido, o que seas un Melville, quien puso en boca de Bartleby, el escribiente, la frase «Prefiero no hacerlo».

Margolles (2014) señaló cuatro condiciones para obtener un buen título: seriedad, claridad al describir el contenido, precisión en las palabras clave y un componente emotivo. 
https://doi.org/10.46744/bapl.201802.006

La relación entre las palabras clave y el título es muy estrecha, como si fueran novios. Esa relación se garantiza si escribes el título desde lo general a lo específico, ya que uno se guía por el título para elegir las palabras clave. $\mathrm{O}$, al revés, si usas las palabras clave para escribir el título. Para esa tarea, una buena ayuda son los tesauros (Jara Casco, 1999).

Otra recomendación es recurrir a contenidos cortos y emotivos. Quienes asignan los títulos a las películas son expertos en eso. $\mathrm{He}$ revisado varios títulos de ese estilo, como «No disparen al turista» (sobre los riesgos del turismo), o "Todo lo que sube cae» (sobre la castaña), o «¿Por quién doblan las campanas?» (sobre los horrores del narcotráfico). Son títulos que, así solos, no dicen mucho, pero si le agregas un subtítulo que remita a un problema concreto llaman la atención. Son títulos cebo, que atraen lectores. Pienso en dos, que les obsequio: «Lo que el viento se llevó. Huracanes en Puerto Rico, 20102017». O «La venganza de Moctezuma. El ají en la comida tradicional de México». Con ellos, un autor logra su propósito: un lector cazado, y entusiasmado por IMRYD. Ahora es tu responsabilidad retenerlo e interesarlo. En esa tarea estás frente a un lector crítico, pero no solo: te acompañan los autores que han escrito sobre el tema y que acudirán a tu auxilio, para ayudarte a desplegar tus argumentos. La escogencia de esos autores va por cuenta tuya. Como en un juego de fútbol improvisado, en el que los dos capitanes autoelegidos escogen los mejores jugadores para su equipo. Tienes buenas guías. Una de ellas es una buena biblioteca y especialmente el Google scholar o académico, donde escriben los especialistas.

\section{La importancia de un buen resumen}

Si el resumen no está bien escrito y no refleja el contenido del artículo, es mejor que no lo leas. No pierdas el tiempo. Un resumen es una suerte de píldora del artículo concreto. Como un juego de habilidades, donde el autor pone a prueba su organización, su orden mental y el uso idóneo del lenguaje. Una ardua tarea en la que el autor debe exponer con claridad el contenido de su artículo en no más de trescientas palabras, menos de la mitad de una página. ¿Te parece poco o mucho? En un resumen 
corto, debes, usando el tiempo verbal del pasado, indicar los objetivos planteados y el método empleado. Si quieres agregar más información, menciona las principales conclusiones y enuncia la discusión sobre el tema (Villagrán \& Harris, 2009).

Los expertos recomiendan escribir el título y el resumen al final del trabajo, aunque aquellos hayan estado, como si fueran aves de rapiña del lenguaje, dando vueltas continuamente sobre el asunto. Parece un contrasentido, pero es así. Hay pocas excepciones. Una de las más notables es la del novelista chileno Roberto Bolaños, fallecido en plena madurez creativa, que ponía el título antes de comenzar a escribir el libro.

\section{Las dificultades para plantear una discusión razonable}

Para todos los autores de libros sobre metodología científica, la escritura de la «discusión» resulta la parte más difícil para un articulista porque requiere de un elevado sentido de la crítica. Allí no se pueden repetir los resultados y nadie da muchas pistas de cómo escribirla. Se trata de interpretar los resultados obtenidos poniéndolos en un contexto más amplio que el del propio artículo. Se trata de criticarse a sí mismo, hablando de las dificultades encontradas y de las limitaciones en el alcance. Aguzar la imaginación hacia lo que significan los hallazgos propios en conexión con los hallazgos de otros, con la mayor objetividad. La discusión se parece mucho a lo que se propone en un artículo científico de «revisión del estado de la cuestión»: empleando la información publicada por otros, que actúa como una plataforma de lanzamiento o un mirador, que nos permita ver más lejos en el horizonte de un problema o de una cuestión. Empinarse, como dice el lema de Google académico, «sobre hombros de gigantes».

Villagrán \& Harris (2009) señalan que la discusión es el corazón mismo del artículo. Allí se interpretan los resultados encontrados en relación con los objetivos planteados y con el estado actual del conocimiento del tema. Aconsejan escribirla en presente. Todo el artículo científico debe ser escrito en pasado, salvo la introducción y la discusión. 
https://doi.org/10.46744/bapl.201802.006

Es, en síntesis, una tarea muy complicada: decir con claridad lo que se ha encontrado o no, dando pistas a otros investigadores para que avancen más allá de donde uno ha llegado. Ese desprendimiento no es una virtud muy extendida entre los seres humanos, y menos entre los científicos. Los científicos son como los cocineros prestigiosos: aman su oficio, pero no sueltan el verdadero secreto de sus grandes creaciones. Los científicos son protagonistas de resentimientos legendarios de los que nos habla con profusión la historia. Escribir la discusión es como el último envión de los escaladores antes de llegar a la cima de la montaña. Lo arduo queda atrás, pero aún falta por hacer el último gran esfuerzo.

\section{La palabra como palanca...}

Ahora, last but no least, ingreso en el terreno de la literatura para examinar cómo la literatura puede ser soporte de la comunicación científica, a pesar de que para muchos es una relación incompatible con la esencia de la ciencia.

Un artículo científico cumple su objetivo cuando comunica información científica con eficacia (es decir, la realiza) y eficiencia (lo hace óptimamente, con el menor esfuerzo). Eso se logra cuando un artículo científico es claro en lo que intenta transmitir. Pero escribir con claridad no es una habilidad innata. Hay que aprenderla y desarrollarla con la práctica, como sucede en todos los oficios. Si quieres comer un buen postre, pídeselo al pastelero, y no al herrero. Para escribir un buen texto, hay que recurrir a los buenos escritores, que se ganan la vida ordenando las palabras, buscándoles la música que encierran, para convertir un texto ordinario en extraordinario. Oír y leer a los grandes escritores es, pues, una buena idea. Con ese propósito acudimos a los consejos de Gabriel García Márquez, Nobel de Literatura 1982; Ernest Hemingway, Nobel 1954; Paul Auster, un novelista estadounidense que será Premio Nobel, y al escritor estadounidense Stephen King, que nunca lo será, a pesar de que se han vendido más de trescientos cincuenta millones de ejemplares de sus cincuenta novelas, de las que se han filmado más de treinta películas y series de TV. Es útil, además, contar con dos destacados académicos del lenguaje. Uno es Steven 
Pinker, neurocientífico y lingüista de la Universidad de Harvard. El otro es Umberto Eco, semiólogo y novelista italiano, autor de El nombre de la rosa, de la que se han vendido varios millones de ejemplares. En suma, una compañía muy recomendable.

Eco (2001) señala «cuatro reglas obvias» para elegir un tema: 1. Que el tema corresponda a tus intereses y gustos. 2. Que las lecturas básicas estén a tu alcance. Ahora es simple: basta con tener una computadora conectada a internet. 3. Que manejes las fuentes que utilizarás. En tu propio idioma, si no manejas otro, y según tu nivel de comprensión y antecedentes personales. 4. Que la metodología a usar esté a tu alcance. Si vives en el Perú, no es aconsejable escribir sobre la cocina tradicional tailandesa o los sismos en Japón.

Pinker (2014) recomienda ubicar al lector desde el principio explicándole cuál es el punto de partida. Informarlo sobre el motivo del viaje para que te acompañe con gusto. Lee, por favor, el poema Itaca de Kavafis. También recomienda que hagas un esfuerzo para escribir con corrección. Que no sientas temor de expresarte como si existiera una policía del lenguaje que vigila cada palabra que escribes. Que comunicarte es un acto social, que lo que expresas es tu responsabilidad, que en esa tarea es lícito emplear algunos atajos. Que no escurras el bulto a la hora de expresarte.

¿Cuáles atajos? King (2001) vino en nuestra ayuda. El recurso más simple para expresarse, dice, es el de la voz pasiva o lenguaje indirecto. Pero eso es un recurso de escritores tímidos, tan reprimidos que filtran cada palabra que expresan. King aconsejó que en vez de escribir, por ejemplo, «La reunión ha sido programada para las siete», escriban «la reunión es a las siete».

Para evitar los atajos, que es una manera de escurrir el bulto al desafío de la escritura, y en lugar de regresar a la escuela primaria para aprender sintaxis y gramática elementales, los grandes escritores recomiendan un recurso que nunca falla: la lectura. Se debe, parodiando al poeta Rainer María Rilke, leer, leer, leer (escribir, escribir, escribir) y cuando uno se canse de leer (o de escribir), se debe 
seguir leyendo (o escribiendo). Es una recomendación con garantía de éxito. La señalaron los escritores más renombrados del planeta: Faulkner, Zola, Rilke, Kafka, Hemingway, Cortázar, García Márquez, Vargas Llosa, Auster, Steinbeck, Proust, Balzac, Saramago, Neruda, Eco, Borges, Carver, Coetzee, Mishima, y muchos otros más. Aunque la lista es finita, parece infinita. Dos palabras más para terminar este párrafo. La primera es sobre José Saramago, el novelista portugués Nobel de Literatura en 1998. Saramago abandonó la escuela en el poblado rural en el que vivía, porque sus padres, campesinos pobres, lo necesitaban para el trabajo agrícola. Después, en la medida en que crecía, pasó por varios oficios: herrero, vendedor de seguros, mensajero, hasta que llegó a una editorial en un periódico, donde se hizo amante de los libros. Leía y leía, sin descanso. Escribió dos novelas que no tuvieron éxito. Decepcionado, dejó de hacer literatura durante veinte años porque, confesó, «no tenía nada que decir». Hasta que un día, sobreponiéndose a su miedo, escribió una novela que le gustó a mucha gente. Y después escribió otra, y otra, hasta llegar, casi sin darse cuenta, al Nobel de Literatura. La segunda palabra prometida es sobre Jorge Luis Borges, el gran escritor argentino que dijo una vez: "Leer es un verbo que no admite el modo imperativo». En verdad, nadie puede obligarte a leer, salvo en tu tiempo de escuela. Leer resulta aburrido si lo haces por obligación y no por placer.

La escritura no es fácil para nadie. No lo es para los científicos ni para los escritores.

Charles Darwin, el científico de la teoría de la evolución, autor de El origen de las especies», confesó que «La vida del naturalista sería dichosa si solo tuviera que observar, sin escribir nunca». El novelista Gabriel García Márquez, autor de Crónica de una muerte anunciada, dijo que una página en blanco es como un ring de boxeo, en el cual uno se cae a golpes con las palabras. Ellas lo golpean a uno, y uno a ellas.

Day (2005), citado con frecuencia en la tarea de la escritura de artículos científicos, señaló que un artículo científico no se hace para entretener, sino para comunicar información científica al lector. Por eso 
debe ser redactado de una manera sencilla, clara y ordenada. «El mejor lenguaje - dice- es el que transmite el sentido con el menor número posible de palabras». Recuerdo que eso lo dijo el jesuita y escritor del Siglo de Oro español Baltasar Gracián, en 1647: «Lo bueno si breve, dos bueno». Day dice, además, que es más importante la organización del material que el talento para escribirlo, y que «la grandilocuencia no tiene cabida en la redacción científica». Pero Day es esclavo de lo que dice. La brevedad no es, empero, sinónimo de claridad, «transmisor de sentido». Se equivoca también cuando afirma que la grandilocuencia, entendida como adorno excesivo del lenguaje, es una característica del lenguaje literario. Un buen escritor, al menos un escritor moderno, no es grandilocuente per se, pues se expresa con suma claridad, con palabras sencillas, sin rebuscamientos, con un estilo donde no sobra ni falta nada. La oscuridad es, con frecuencia, propia del discurso científico, salvo el de la etnología y de los divulgadores de la ciencia. Nietzsche decía que hay filósofos que se esmeran en buscar palabras rebuscadas para hacer que las aguas cristalinas se vuelvan turbias y parezcan más profundas de que realmente son.

La humildad de un científico lo debe llevar a leer a grandes escritores para aprender a transmitir con claridad sus importantes descubrimientos científicos. En eso era un maestro el médico endocrino español Gregorio Marañón, que escribía ciencia como si fuera un ensayo literario. Los farmacólogos Ferriols \& Ferriols (2005), autores de manuales para escribir artículos científicos, dicen que el objetivo de estos es «comunicar los hallazgos derivados del proceso de investigación». Para hacerlo, recomiendan emplear un lenguaje preciso, sin ambigüedades, claro, que se entienda fácilmente, con palabras sencillas, no rebuscadas. Un lenguaje conciso, utilizando las palabras justas. Para ellos, escribir bien no es una habilidad innata sino aprendida con la práctica. Y los mejores maestros en esa tarea son los grandes literatos.

Si quieres comunicar bien algo, y que tu lector comprenda, tienes forzosamente que escribir bien. En ese campo no hay diferencias de géneros académicos, de ciencias sociales o ciencias experimentales. No hay excusa que valga. O se escribe bien para hacerse entender o se escribe mal y nadie 
https://doi.org/10.46744/bapl.201802.006

nos entiende. Aunque se debe reconocer que no es lo mismo escribir ciencia en el siglo XIX que hacerlo hoy (Bazerman, 1994), y aceptar que hay diversidad de géneros discursivos, porque cada esfera de la práctica tiene su propia manera de decir su discurso (Bakhtin, 1982).

Escribir bien es como participar en una carrera de obstáculos con una mochila al hombro (esa mochila pesa y te limita: es lo que debes decir). Para llegar con éxito a la meta, hay que evitar los tropiezos. Ferriols \& Ferriols (2005) aconsejan no abusar del empleo del gerundio (observando, examinando, planteando), en vez de decir se observaron, se examinó, se planteó). No usar en exceso frases vacías (decir «...Dado que las evidencias muestran que..., en lugar de así pues, y en base tanto a las evidencias observadas). Recomiendan evitar la negación doble (en lugar de En el texto no aparece ninguna revista peruana citada, decir En el texto no se citan revistas peruanas). No se debe olvidar algo muy importante: en cada párrafo debe haber concordancia en el tiempo verbal y coherencia en el uso del singular o del plural.

La escritura es un acto reglamentado o condicionado por el uso de convenciones lingüísticas y textuales (Castelo, 2009; Capomagi, 2013). Para sortear esos condicionamientos tenemos que respetar esas convenciones. Una de ellas es la de citar las voces de los otros que hemos usado dentro de nuestro propio discurso. La escritura es más que un canal para transmitir conocimientos y datos. El lenguaje expresa la estrecha relación existente entre el pensamiento y el conocimiento, el pensamiento y la acción (Cassany, 2006). En este sentido, sujeto y verbo son como una pareja de enamorados que andan siempre juntos (King, 2001).

Los médicos Villagrán \& Harris (2009) ofrecen algunos consejos para mejorar la redacción de los artículos científicos. Se trata de una suerte de decálogo. 1. Desarrolla una idea en cada párrafo. Si usas dos párrafos. 2. Evita el uso de las mayúsculas al inicio de términos que indiquen conceptos o categorías como Ética, Verdad, Justicia, Universidad, Biología, etc. 3. Evita el uso de expresiones en infinitivo. 4. No enuncies lo que vas a decir. Dilo de una vez. En vez de «Podemos 
considerar que la muestra no es claramente representativa», di: «la muestra no es representativa», y explica por qué. 5. No hagas oraciones mayores de treinta palabras, entre punto y punto. 6. No uses el verbo en pretérito pasado, como «Ha obtenido», escribe: «obtuvo». 7. No uses frases en lengua pasiva, como "La enfermedad es así percibida como ocasionada por...», en vez de "La enfermedad se percibe como....» 8. Usa correctamente los tiempos verbales para el artículo: el pasado para el resumen, la metodología y los resultados, y el presente para la introducción y la discusión. 9. No hagas reiteraciones enfáticas. Ve al grano. No escribas, por ejemplo: reitero, en vez de reitero. O prever de antemano, porque prever y antemano es lo mismo. 10. No olvides que los primeros lectores de tu artículo son el editor y el revisor, y lo primero que leen es el título, el perfil académico y el resumen.

Jara Casco (1999) y Slafer (2009) dan el mismo consejo: no des vuelta, anda al grano. Slafer recomienda al autor hacerse tres preguntas a la hora de escoger la revista en donde publicar: isirve para algo mi artículo?, ¿podrá interesarle mi artículo a esa revista?, ¿he escrito mi artículo de manera clara y concisa? Es una buena costumbre revisar la estructura de la posible revista en donde publicar, examinar algunos de sus contenidos, lee algunos resúmenes. Otra buena costumbre es apartarte una semana de tu artículo terminado. Tu artículo te parecerá ajeno y lo verás más críticamente.

Emplear oraciones breves es clave. Grandes escritores como Faulkner, Hemingway y Kafka rindieron culto a la frase breve, no muy larga. Es un estilo de escritura que no todos los escritores comparten. La frase corta beneficia la claridad del texto. Te permite ordenar mejor la lectura y la comprensión de la idea que expresas. El uso de la oración larga es más difícil, y solo la pueden emplear con éxito algunos escritores excepcionales como Cortázar, porque es difícil mantener el «aire» de la frase, como si uno dejara de respirar, sin que se pierda el sentido de lo que uno quiere decir. ¿Ves? Hice una frase larga, usando varias comas, y casi me asfixié.

Daré dos ejemplos de escritores que están en su mejor momento. Uno es la española Elvira Lindo (2015). El otro es el estadounidense 
Paul Auster (2012). Te recomiendo, tras leer cada texto, examinar los pensamientos contenidos.

Lindo (2015: 19): "Aun así, no quiero escribir aquí los años que cumplo. No me da la gana. No los siento. No son míos. No he llegado a ningún sitio, ni quiero. No soy una mujer madura, tampoco un proyecto de vieja. Y al que me diga mañana que me conservo muy bien le tomaré una manía inconfesable durante un tiempo».

Auster (2012: 79): «Yo tenía ocho años. En aquel momento de mi vida, nada me importaba más que el béisbol. Mi equipo era el New York Giants, y seguía las actividades de aquellos hombres de gorra naranja y negro. Incluso ahora, al recordar a ese equipo que ya no existe, soy capaz de recitar los nombres de casi todos los jugadores».

Busca siempre la ayuda de grandes escritores, en particular cuando sientas que te atascas tratando de escribir algo que no te sale. Lee buenos artículos cortos como los del español Antonio Muñoz Molina, novelista y catedrático de la lengua española, que escribe en El País, de Madrid. Después de leer, por ejemplo, El Viejo y el Mar, de Hemingway, sentirás que tu escritura se vuelve más suave, como si le hubieran untado mantequilla. No te sientas menos por pedir ayuda a esos grandes. Recuerda el refrán: «A quien a buen árbol se arrima buena sombra lo cobija».

Un último consejo. Lee, para practicar, estos seis libros breves, que son modelos de buena escritura: El viejo y el mar (E. Hemingway), El coronel no tiene quien le escriba (G. García Márquez), La metamorfosis (Kafka), Muerte en Venecia (T. Mann), Pedro Páramo (J. Rulfo), El cuaderno rojo (P. Auster).

\section{Discusión}

Este artículo se basó en dieciocho citaciones. Pocas, relativamente pocas para un tema como este, sobre el cual existe una abundante producción bibliográfica. La lista de publicaciones citadas está desactualizada: solo 
seis son de los últimos cinco años. La razón es muy simple. Hay muchos libros relacionados con el tema, pero todos se repiten. No aporto, además, conocimientos originales sobre la cuestión. Actúo solo como facilitador de apenas una idea: la necesidad que tienen tanto los científicos como los escritores de exponer con claridad sus hallazgos o sus ideas. Una necesidad compartida por ambos, y un propósito del que ambos se benefician. El escritor que lee ciencia divulgativa bien escrita se beneficia extrayendo de allí personajes, escenas y acciones para sus novelas. El científico que lee buena literatura puede escribir los hallazgos de su ciencia con claridad y amenidad, multiplicando sus lectores y haciendo realidad el hecho de compartir sus descubrimientos con mucha gente, tal como hicieron en su tiempo el morfólogo y enunciador de la teoría del color Johann Wolfgang Goethe, el geógrafo, astrónomo y naturalista Alexander von Humboldt, el poeta y dramaturgo Maurice Materlinck, el matemático Lewis Carroll, el filósofo H.G. Wells, el bioquímico Isaac Asimov, el médico Arhur Conan Doyle, el astrónomo Carl Sagan, el filólogo J.R.R. Tolkien, el físico Arthur C. Clarke, el biólogo Edward O. Wilson, el físico teórico Stephen Hawkings, el neurobiólogo Steve Pinker, y más cerca de nosotros el físico Ernesto Sábato, el matemático Nicanor Parra, y otros (desgraciadamente no muchos) esclarecidos maestros de su ciencia. 


\section{BIBLIOGRAFÍA}

AUSTER, P. (2012). El Cuaderno Rojo. Barcelona: Seix Barral.

BAKHTIN, M. (1982). Estética de la creación verbal. Madrid: Siglo XXI Editores.

BAZERMAN, C. (1994). Systems of genres and the enactment of social intentions. En: Freedman, A.; Medway, P. Genre and The New Rhetoric, London: Taylor \& Francis, 79-101, 1994.

BRAILOVSKY, D. (2010). Cincuenta expresiones usuales del lenguaje académico. Buenos Aires: UCES.

CAPOMAGI, D. (2013). La escritura académica en el aula universitaria. Revista de Educación y Desarrollo, 25.

CASSANY, D. (2006). Taller de textos: leer, escribir y comentar en el aula. Barcelona: Paidós.

CASTELLÓ, M. (2009). Aprender a escribir textos académicos, ¿copistas, escribas, compiladores o escritores? en: Pozo, J.I.; Pérez E., M.P. (coord.). La psicología del aprendizaje universitario: de la adquisición de conocimientos a la formación de competencias. Madrid: Morate, 120-133.

DAY, R.A. (2005). ¿Cómo escribiry publicar artículos científicos? Washington, D.C.: Organización Panamericana de la Salud, 2005.

DI MARCO, M.; PANDZIK, N. (2013). Atreverse a escribir. Buenos Aires: Sudamericana.

ECO, U. (2001). ¿Cómo se hace una tesis? Barcelona: Editorial Gedisa.

FERRIOLS, L. R.; FERRIOLS, L.F. (2005). Escribir y publicar un trabajo científico $V$. 
https://doi.org/10.46744/bapl.201802.006

JARA CASCO, E. (1999). La selección del título en el artículo científico. Revista Cubana Medicina General Integral, 15, 3, 342-345.

KING, S. (2001). Mientras escribo. Barcelona: Plaza \& Janéz.

LINDO, E. (2015). Noches sin dormir. Barcelona: Seix Barral.

MARGOLLES, P. (2014). El arte de escribir títulos de artículos científicos memorables. NeoScientia, Blog, 23.02.

PINKER, S. (2014). The Sense of Style: The Thinking Person' Guide in Writing in the 21sth Century. New York: Viking Penguin.

SLAFER, G. A. (2009). ¿Cómo escribir un artículo científico? Revista de Investigación en Educación, 6, 124-132.

VILLAGRÁN, A. y HARRIS, P. (2009). Algunas claves para escribir correctamente un artículo científico. Revista Chilena de Pediatría, 80 (1), 70-78. 